\title{
Koebner phenomenon in vitiligo after suction blister epidermal grafting
}

\author{
Wei Liu MD, Dong-Lai Ma MD PhD
}

— Cite as: CMAJ 2019 September 3;191:E968. doi: 10.1503/cmaj.190070

A

14-year-old girl who had nonsegmental vitiligo on her face, trunk and extremities for more than 1 year presented for consultation on new areas of abdominal depigmentation. Two months earlier, she had undergone suction blister epidermal grafting. The donor sites were chosen from unaffected areas on her abdomen, and the recipient site was a depigmented patch on her face. Soon after the procedure, new depigmented macules and patches with a diameter of approximately $1 \mathrm{~cm}$ developed at previously unaffected donor sites (Figure 1). We diagnosed Koebner phenomenon secondary to suction blister epidermal grafting, and we prescribed oral prednisone $30 \mathrm{mg} / \mathrm{d}$ and topical pimecrolimus and halometasone. Two months later, the lesions had become less visible.

Suction blister epidermal grafting has been reported as an effective treatment of vitiligo. ${ }^{1}$ Donor sites are selected from an unaffected area, then both donor sites and recipient sites are attached to the blister-forming device. Round blisters with a diameter of $5-8 \mathrm{~mm}$ form several hours later, and skin is transferred between sites.

Koebner phenomenon is defined as "the development of lesions at sites of distinctively traumatized uninvolved skin of patients with cutaneous diseases."2 A classification system for Koebner phenomenon in vitiligo has been proposed: type 1 , diagnosed based on a patient's history; types $2 \mathrm{~A}$ and $2 \mathrm{~B}$, based on clinical examination (2A: lesions on friction areas; $2 \mathrm{~B}$ : linear, artifactual lesions); and type 3 , based on experimental induction. ${ }^{3}$ In the case of our patient, Koebner phenomenon type 2B resulted from suction blister epidermal grafting. Clinicians may wish to consider the possibility of Koebner phenomenon when performing procedures when vitiligo is active.

\section{References}

1. Gou D, Currimbhoy S, Pandya AG. Suction blister grafting for vitiligo: efficacy and clinical predictive factors. Dermatol Surg 2015;41:633-9.

2. Miller RA. The Koebner phenomenon. Int J Dermatol 1982;21:192-7.

3. van Geel N, Speeckaert R, Taieb A, et al.; VETF members. Koebner's phenomenon in vitiligo: European position paper. Pigment Cell Melanoma Res 2011;24: 564-73.

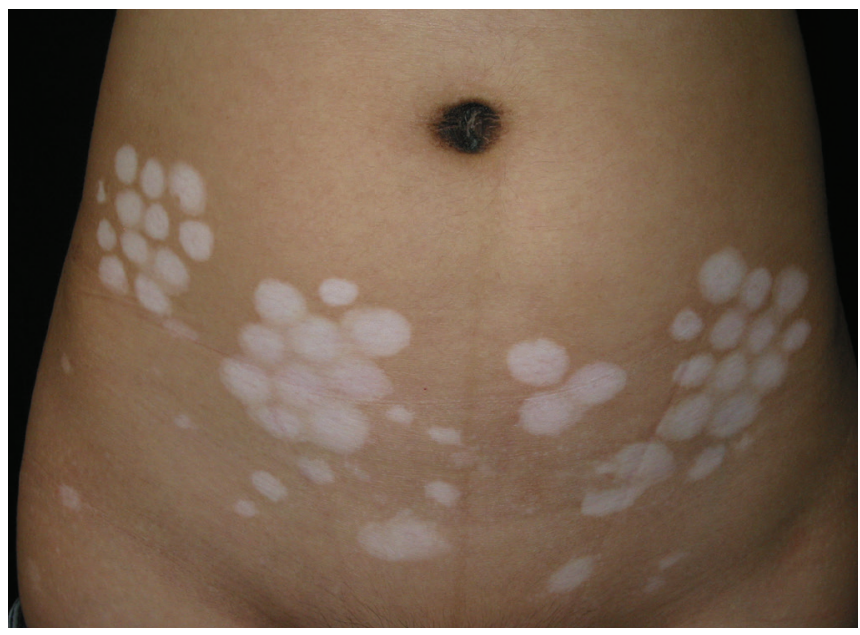

Figure 1: Multiple nummular depigmented macules in the donor areas of the abdomen of a 14-year-old girl with vitiligo.

Competing interests: None declared

This article has been peer reviewed.

The authors have obtained patient consent.

Affiliation: Department of Dermatology (Liu, Ma), Peking Union Medical College Hospital, Chinese Academy of Medical Sciences and Peking Union Medical College, Beijing, China

Correspondence to: Dong-Lai Ma, mdonglai@sohu.com

Clinical images are chosen because they are particularly intriguing, classic or dramatic. Submissions of clear, appropriately labelled high-resolution images must be accompanied by a figure caption. A brief explanation (300 words maximum) of the educational importance of the images with minimal references is required. The patient's written consent for publication must be obtained before submission. 